\title{
Rapid detection of trace amounts of surfactants using nanoparticles in fluorometric assays $\dagger$
}

\author{
Harri Härmä, ${ }^{* a}$ Susana Laakso,${ }^{a}$ Sari Pihlasalo, ${ }^{a}$ Pekka Hänninen, ${ }^{a}$ \\ Bertrand Faure, ${ }^{b}$ Subhasis Rana ${ }^{b c}$ and Lennart Bergström ${ }^{b}$
}

\author{
Received 3rd July 2009, Accepted 15th October 2009 \\ First published as an Advance Article on the web 16th November 2009 \\ DOI: $10.1039 / \mathrm{b} 9 \mathrm{nr00172g}$
}

\begin{abstract}
Rapid microtiter assays that utilize the time-resolved fluorescence resonance energy transfer or quenching of dye-labeled proteins adsorbed onto the surfaces of polystyrene or maghemite nanoparticles have been developed for the detection and quantification of trace amounts of surfactants at concentrations down to $10 \mathrm{nM}$.
\end{abstract}

Surfactants find widespread use as dispersants, emulsifiers, detergents, fabric softeners and wetting agents in many household items and industrial products and processes. ${ }^{1}$ The environmental impact and toxicity of various surfactants, and current legislation, require that the amount of surfactants released into the sewer system is minimized and that the concentrations in rivers and lakes are maintained at low levels. ${ }^{2}$ As a consequence, the use of closed processes is rapidly increasing in those industries consuming large quantities of surfactants, e.g. the paper and pulp industries. Closed processes are sensitive to accumulation and rapid fluctuations in surfactant concentrations. Hence, the strict regulations for waste water release and the rapid development and introduction of closed processes call for novel techniques that allow fast and simple detection of low concentrations of surfactants.

Surfactants can be detected and analyzed with a range of techniques depending on their composition. ${ }^{3}$ While ionic surfactants can be readily quantified by surfactant-selective electrodes, ${ }^{4 a}$ methods capable of also detecting non-ionic surfactants are more complex and involve HPLC, ${ }^{4 b}$ gas chromatography, ${ }^{4 c}$ capillary electrophoresis, ${ }^{4 d}$ contact angle measurement, ${ }^{4 e}$ refractometric analysis, ${ }^{4 f}$ and fluorescence techniques. ${ }^{4 g}$ However, many of these techniques suffer from low sensitivity, and time-consuming pre-concentration and analysis procedures making them impractical for rapid detection.

We present here a novel technique where a combination of nanoparticle - fluorescently-tagged protein pairs are utilized for the detection of very low surfactant concentrations in a fast, easy-to-use and inexpensive assay setup. The method builds on previous work where nanoparticle-based time-resolved fluoroassays of proteins at low concentrations were developed. ${ }^{5}$ Fig. 1 illustrates the two different assay principles applied to detect surfactants in solution. In the europium(III) (Eu) chelate-impregnated nanoparticle

${ }^{a}$ Laboratory of Biophysics and Medicity, University of Turku, Tykistökatu 6A, FI-20520 Turku, Finland.E-mail: harri.harma@utu.fi

${ }^{b}$ Materials Chemistry Research Group, Dept. of Physical, Inorganic and Structural Chemistry, Stockholm University, SE-106 91 Stockholm, Sweden

'Sensor and Actuator Division, Central Glass \& Ceramic Research Institute, Kolkata 700 032, India

$\dagger$ Electronic supplementary information (ESI) available: Experimental details and Fig. S1 and S2. See DOI: 10.1039/b9nr00172g
(Eu-PSCOOH) sensor, the fluorescence resonance energy transferred from the nanoparticle to the Alexa Fluor 680 acceptor label coupled to gammaglobulin (IgG-Alexa680) is monitored at $730 \mathrm{~nm}$. The decrease of the fluorescence intensity is related to displacement of the labeled protein by a surfactant (Fig. 1A-B). In the maghemite $\left(\gamma-\mathrm{Fe}_{2} \mathrm{O}_{3}\right)$ particle sensor, the fluorescence signal of dipyrrylmethene$\mathrm{BF}_{2}$ 530-labeled BSA at $572 \mathrm{~nm}$ is strongly quenched when the $\mathrm{BF}_{2}$ 530-labeled protein is adsorbed onto the nanoparticle surface (Fig. 1C-D). Hence, displacing or prohibiting the adsorption of the labeled protein to the maghemite surface by a surfactant reduces the quenching and results in an increase in the fluorescent intensity.

The commercially available Eu(III)-doped carboxylated polystyrene spheres ${ }^{6}$ produced by an emulsion polymerization route are relatively monodisperse with an average diameter of $73 \mathrm{~nm}$ (Fig. 2A). The surface chemistry of these particles is dominated by ionizable

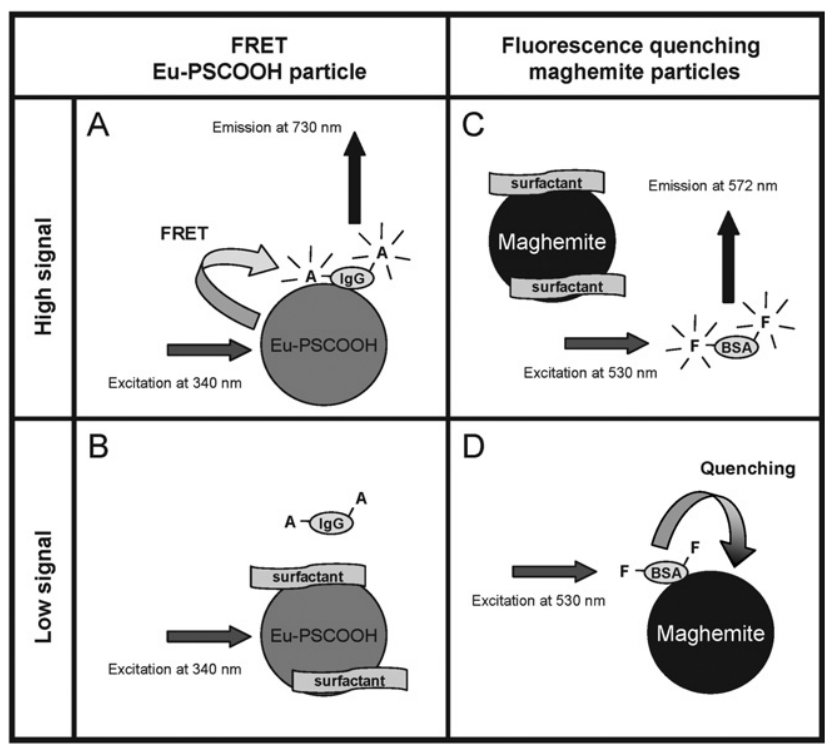

Fig. 1 Schematic illustration of the fluorescence detection principles and the effect of surfactant displacement for the two different nanoparticlefluorescent protein pairs. The left side shows the fluorescence resonance energy transfer (FRET) between the europium(III) (Eu) chelate-impregnated nanoparticle and the adsorbed Alexa Fluor 680 acceptor label coupled to gammaglobulin ( $\operatorname{IgG})(\mathrm{A})$. The surfactant prevents the adsorption of the labeled IgG and low FRET signal is monitored (B). The right side illustrates the quenching of the fluorescence of dipyrrylmethene$\mathrm{BF}_{2} 530$ conjugated to bovine serum albumin (BSA) when adsorbed on the surface of maghemite nanoparticles (D). The fluorescence emission is detected in solution as the surfactant replaces the labeled BSA (C). 

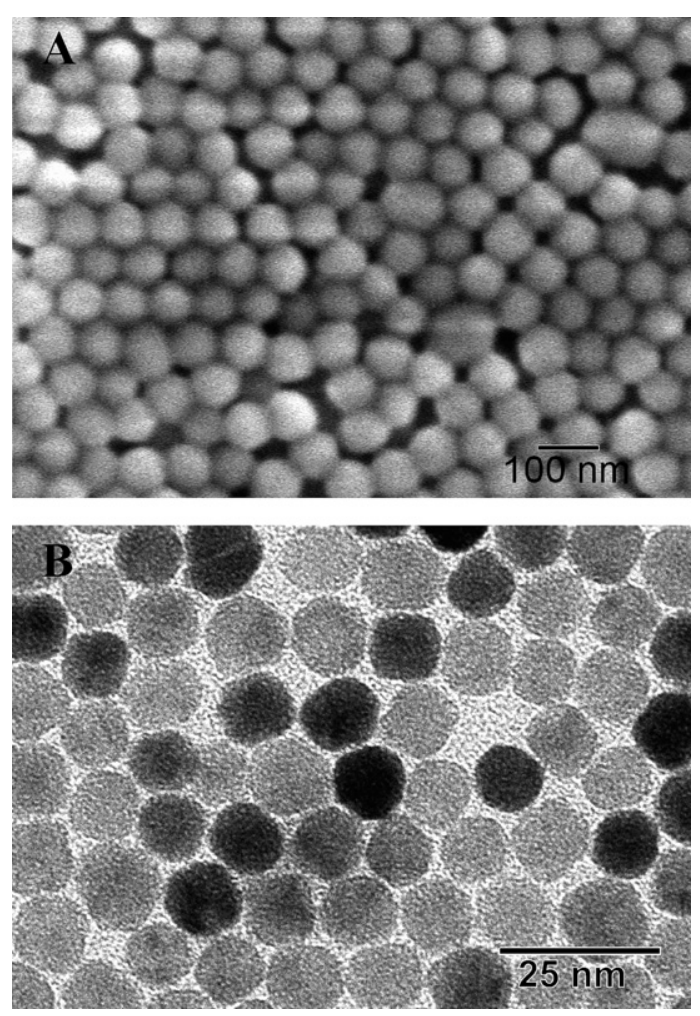

Fig. 2 SEM image of (A) the Eu-PSCOOH particles and (B) TEM image of the water-dispersible iron oxide nanocrystals.

carboxylic groups, in addition to sulfates, that render a negative surface charge over a relatively wide $\mathrm{pH}$ range. ${ }^{7} \mathrm{Fig}$. $2 \mathrm{~B}$ shows that the synthesized maghemite nanocrystals have a very well-defined spherical morphology and a narrow size distribution with an average diameter of $13 \mathrm{~nm}$. A controlled leaching procedure was able to remove the oleic acid capping yielding water-dispersable hydrophilic maghemite nanocrystals (Fig. S1 $†$ ). Iron oxides like maghemite have ionizable amphoteric surface hydroxyl groups $(\mathrm{Fe}-\mathrm{OH})$. The point of zero charge, i.e. the $\mathrm{pH}$ where the total surface charge is zero, is around $\mathrm{pH} 7$; hence, the maghemite nanoparticles are expected to be positively charged at $\mathrm{pH} 4$ in the assay buffer. ${ }^{7}$

The nanoparticle sensor assays were carried out in a $5 \mathrm{mM}$ citratephosphate buffer at $\mathrm{pH}$ 4.0. The surfactant-containing solution was mixed with the nanoparticles $\left(2 \times 10^{7}\right.$ Eu-PSCOOH or $4 \times 10^{11}$ maghemite nanocrystals) and $5 \mu \mathrm{L}$ Alexa Fluor 680-labeled gammaglobulin $(1.9 \mathrm{nM})$ or $20 \mu \mathrm{L}$ of dipyrrylmethene- $\mathrm{BF}_{2}$ 530-labeled BSA $(5.9 \mathrm{nM})$. The dispersion was mixed well, and transferred to a 96-well microtiter plate where the fluorescence measurements were performed within less than $5 \mathrm{~min}$.

The nanoparticle sensors were evaluated by measuring the response to four different surfactants; the cationic surfactant cetyl trimethylammonium bromide (CTAB), the anionic surfactant sodium dodecyl sulfate, (SDS), and two different non-ionic surfactants (Tween 20 and Triton X-100). Fig. 3 shows that both the Eu-PSCOOH/IgG-Alexa680 and the maghemite/BF 2 530-BSA sensors resulted in a distinct response for all the investigated surfactants. The reproducibility was relatively good with an average coefficient of variation of $10.7 \%$ (measurements performed thrice).

The Eu-PSCOOH/IgG-Alexa680 sensor (Fig. 3A) was able to detect the cationic surfactant, $\mathrm{CTAB}$, at concentrations as low as
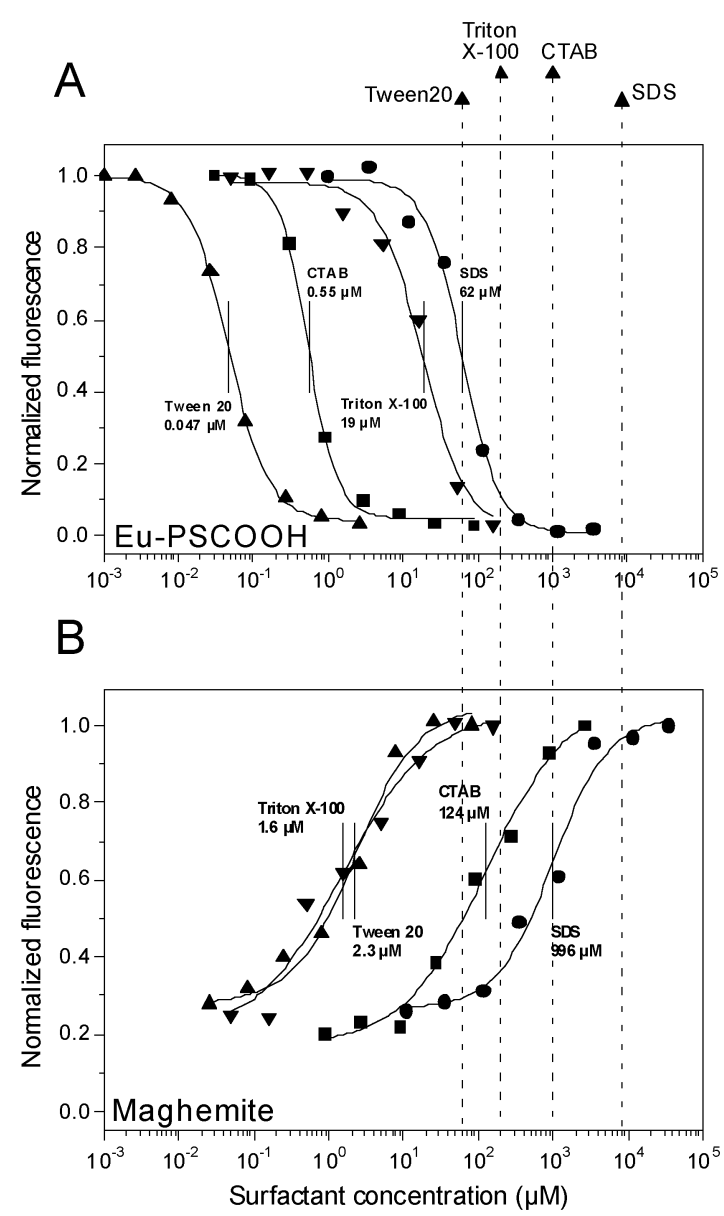

Fig. 3 Fluorescence detection of surfactants, Tween 20 ( $\boldsymbol{\Delta})$, Triton X-100 ( $\boldsymbol{\nabla})$, CTAB ( $\mathbf{\square})$ and SDS ( $)$. (A) Addition of surfactants results in a decrease in the FRET signal due to the displacement of the Alexa Fluor $680-\mathrm{IgG}$ from the Eu-PSCOOH nanoparticles by the surfactants; $\mathrm{B})$ addition of surfactants results in an increase in the fluorescence due to the displacement of dipyrrylmethene- $\mathrm{BF}_{2} 530$-BSA by surfactants from the surfaces of maghemite nanoparticles. The critical micelle concentrations of the surfactants in water have been indicated with dash lines and the $\mathrm{EC}_{50}$ values of the calibration curves are given.

$200 \mathrm{nM}$, which compares favorably with previously reported detection levels using surfactant-specific electrodes. ${ }^{8}$ However, this system is less sensitive to the anionic surfactant, SDS, with a detection level around $20 \mu \mathrm{M}$. It is not surprising that the detection limit for CTAB is 100 times lower compared to SDS considering the negative surface charge of the Eu-PSCOOH nanoparticles which provides an electrostatic barrier for SDS adsorption. We find that the non-ionic surfactant Tween 20 can be detected at concentrations as low as $10 \mathrm{nM}$ using the Eu-PSCOOH/IgG-Alexa680 sensor. Indeed, the low volumes needed in the microtiter fluorescent assays and the very high sensitivity correspond to detection levels as low as $2 \mathrm{ng}$ for non-ionic surfactants (Tween 20) and $25 \mathrm{ng}$ for ionic surfactants (CTAB). To our knowledge, this is the lowest detection limit for non-ionic surfactants that has been reported.

The maghemite/ $\mathrm{BF}_{2}$ 530-BSA sensor (Fig. 3B) was also able to detect the cationic and anionic surfactants at concentrations more than one order of magnitude lower than their critical micelle concentration in water. However, the detection limit of the 
maghemite sensor for the ionic surfactants was higher compared to the Eu-PSCOOH/IgG-Alexa680 sensor. The very large difference in sensitivity for the cationic surfactant between the maghemite and the $\mathrm{Eu}-\mathrm{PSCOOH}$ sensors suggests that the negative charge on the Eu-PSCOOH particles plays a significant role in promoting the CTAB adsorption and subsequent displacement of the IgGAlexa680, while the positive charge of the maghemite nanoparticles is apparently not sufficient to enhance the competitive adsorption of the anionic surfactant SDS. On the other hand, the maghemite/ $\mathrm{BF}_{2}$ 530-BSA sensor was able to detect the non-ionic surfactant Triton $\mathrm{X}-100$ at a much lower concentration $(0.2 \mu \mathrm{M})$, compared to the Eu-PSCOOH/IgG-Alexa680 sensor $(40 \mu \mathrm{M})$.

The response of the nanoparticle-protein pair to a surfactant may be related to a number of parameters such as the surface charge density of the nanoparticle, the charge of the protein-dye complex and the hydrophobicity/hydrophilicity of the particle surface, the protein-dye complex and the surfactant. ${ }^{1}$ The availability of nanoparticles of different compositions and tunable surface properties, ${ }^{9}$ together with the range of fluorescently tagged proteins already developed for biomedical assays, enables the development of numerous nanoparticle-protein pairs that can be optimized for the detection of specific surfactants. It is also possible that combinatorial analysis of the response of several different nanoparticle-protein systems could provide a "fingerprint" to identify the surfactant in detail. $^{10}$

Further proof for the displacement-based mechanism of the nanoparticle/dye-labeled protein sensors was demonstrated in control experiments, which showed that the addition of surfactants to Eu-PSCOOH dispersions containing no dye-labeled proteins resulted in a negligible decrease of the fluorescence intensity (Fig. S2 $\dagger$ ).

In summary, we have shown how competitive adsorption between surfactants and dye-labeled proteins to the surfaces of nanoparticles can be utilised to detect trace amounts of surfactants in fluorometric assays. The technique may be applied to both a FRET-based system where the fluorescence intensity is decreased due to the displacement of the fluorescently-tagged protein from the europium(III) nanoparticle surfaces by a surfactant, and to a quenched system where the surfactant-dependent displacement of the labeled proteins form the nanoparticle surfaces results in an increase in the fluorescence intensity. The FRET-system consisting of Eu(III)-doped PSCOOH nanoparticle exhibited the lowest limit of detection for most of the studied surfactants, except for the non-ionic surfactant Triton X-100, for which the quenched system consisting of maghemite nanoparticles showed the lowest detection limit. The non-ionic surfactants, which are undetectable with electrode-based methods, could be measured at concentrations as low as $10 \mathrm{nM}$ (Tween 20) without any pre-concentration step. The non-ionic and cationic surfactants could be detected at concentrations more than 1000-fold below their critical micelle concentration. The combination of the novel nanoparticle sensors with existing microtiter plate fluorometric instrumentation could find use as a versatile and rapid multi-task probe for the detection of trace amounts of various types of surface-active molecules in process fluids, of importance in the chemical, pharmaceutical and paper-making industry.

\section{Acknowledgements}

This work was supported by the Finnish Funding Agency for Technology and Innovation (Tekes) and the Swedish Research Council (VR). Anwar Ahniyaz and Bernd Wicklein are thanked for their contribution to the maghemite nanoparticle synthesis and phase transfer procedure; Zoltan Bacsik is acknowledged for assistance with FTIR.

\section{References}

1 B. Jönsson, B. Lindman, K. Holmberg and B. Kronberg, in Surfactants and Polymers in Aqueous Solution, John Wiley \& Sons Ltd, Chichester, 1998.

2 U. Zoller, in Handbook of Detergents, Part B: Environmental Impact, Surfactant Science series, ed. Uri Zoller, Marcel Dekker, New York, 2004, vol. 121.

3 T. Schmitt, in Analysis of Surfactants, Surfactant Science Series, Marcel Dekker, New York, 2nd edn, 2001, vol. 96.

4 (a) J. Sánchez and M. del Valle, Crit. Rev. Anal. Chem., 2005, 35(1), 1529; (b) T. Reemtsma, J. Chromatogr., A, 2003, 1000, 477; P. LaraMartín, A. Gómez-Parra and E. González-Mazo, TrAC, Trends Anal. Chem., 2008, 27, 684; (c) F. Ventura and P. de Voogt, in Analysis and Fate of Surfactants and the Aquatic environment, ed. T. P. Knepper, D. Barceló and P. de Voogt, Elsevier, 2003, vol. 40, (ch. 2), pp. 51; (d) K. Heinig, C. Vogt and G. Werner, J. Chromatogr., A, 1996, 745, 281; K. A. Oudhoff, P. J. Shoenmakers and W. Th. Kok, J. Chromatogr., A, 2003, 985, 479; D. Norton and S. A. Shamsi, Anal. Chem., 2007, 79, 9459; (e) T. C. Kaufmann, A. Engel and H. W. Rémigy, Biophys. J., 2006, 90, 310; (f) P. Strop and A. T. Brunger, Protein Sci., 2005, 14, 2207; $(g)$ C. A. Lucy and J. S. W. Tsang, Talanta, 2000, 50, 1283.

5 H. Härmä, L. Dähne, S. Pihlasalo, J. Suojanen, J. Peltonen and P. Hänninen, Anal. Chem., 2008, 80, 9781; S. Pihlasalo, J. Kirjavainen, P. Hänninen and H. Härmä, Anal. Chem., 2009, 81, 4995.

6 H. Härmä, T. Soukka and T. Lövgren, Clin. Chem., 2001, 47, 561.

7 R. J. Hunter, in Introduction to Modern Colloid Science, Oxford University Press, 2nd edn, 2001.

8 D. Madunic-Cacic, M. Sak-Bosnar, O. Galovic, N. Sakac and R. Matesic-Puac, Talanta, 2008, 76, 259.

9 Y. Yin and A. P. Alivisatos, Nature, 2005, 437, 664; J. Park, J. Joo, S. G. Kwon, Y. Jang and T. Hyeon, Angew. Chem., Int. Ed., 2007, 46(25), 4630.

10 P. Ciosek and W. Wroblewski, Analyst, 2007, 132, 963. 